\title{
Diagnostik und Klassifikation der distalen Radiusfraktur - Die Grundlage der Behandlungsstrategie
}

\author{
Markus Schubert, Andreas Dávid
}

\section{Zusammenfassung}

Die distale Radiusfraktur ist eine der häufigsten Bruchformen und betrifft insbesondere Adoleszente und ältere Menschen. Die Standarddiagnostik umfasst neben der klinischen Untersuchung die Röntgenaufnahme in 2 Ebenen, welche bei spezifischen Fragestellungen durch eine CT- oder MRT-Untersuchung des Handgelenks ergänzt wird. Den Goldstandard zur Beurteilung der intrinsischen Bänder stellt die Handgelenksarthroskopie dar, die unter therapeutischem Ansatz erfolgen sollte. Das Ziel der Diagnostik ist eine eindeutige Klassifikation der Fraktur, wonach eine adäquate Therapie eingeleitet werden kann. Ein gutes System zur Einteilung distaler Radiusfrakturen sollte deswegen beschreibend und gleichzeitig auch voraussagend sein und die Auswahl der geeigneten Behandlungsform wie geschlossene oder offene Reposition, transkutane oder offene Osteosyntheseverfahren ermöglichen. Die Arbeit soll einen Überblick über die diagnostischen Standards und die aktuellen Frakturklassifikationen geben und deren Stellenwert im Gesamtbehandlungskonzept der distalen Radiusfraktur herausstellen. Am weitesten verbreitet ist die Klassifikation der AO unter Berücksichtigung der Stabilitätskriterien nach Jupiter.
Initial Diagnosis and Classification of Distal Radial Fractures A Guideline for Further Treatment

Distal radial fractures are common injuries with a high prevalence among adolescent and elderly osteoporotic women. Besides the clinical examination in most cases the diagnosis can be confirmed by radiographs in two plains. The visualisation of a complex fragmentation of the distal radial articular surface is improved by CT-scan, MRI should be regarded as an option to identify suspected injuries to the intrinsic ligaments. To detect ligament injuries of the wrist, the arthroscopy is the gold standard and should be performed with the intention to treat. The aim of the diagnostic process is to clearly identify and classify the fracture before the adequate treatment is initiated. The systems used to classify the displaced distal radial fractures are supposed to ensure the 3-dimensional assessment of the fracture and provide a high inter- and intra-observer agreement. This article summarises the diagnostic procedures and established classifications. Most common is the Müller-AO classification, the stability is evaluated according to Jupiter's criteria.

\section{Epidemiologische Betrachtung}

Die handgelenksnahen Frakturen der Speiche sowie Verletzungen in Kombination mit einem Bruch der körperfernen Elle stellen eine der häufigsten Bruchfor-

OP-JOURNAL 2012; 28: 228-233

(c) Georg Thieme Verlag KG Stuttgart · New York DOI http://dx.doi.org/10.1055/s-0032-1327995 men des Menschen dar. Bis zu 25\% aller Frakturen des Erwachsenenalters betreffen diese Region. Besonders häufig sind Kinder im Alter von 6-10 Jahren betroffen, wobei in der Gruppe der Adoleszenten die männlichen Patienten überwiegen [3].

Dies ergibt sich aus der bislang noch bestehenden Dominanz der männlichen Sportler in Risiko- und Kontaktsport- arten. Die meisten Frakturen im jugendlichen Alter ereignen sich im Rahmen von Rasanztraumata, Stürzen aus großer Höhe und Sportunfällen. In den letzten Jahren ist es insbesondere in der Gruppe der 10-14-Jährigen zu einem Anstieg der Inzidenz im Rahmen von Sportverletzungen gekommen. Die Unfälle ereignen sich gehäuft im Rahmen des Schulsports beim Turnen und Fußballspielen [5].

Ein 2. Gipfel in der Altersverteilung findet sich zwischen dem 6. und 8. Lebensjahrzehnt. In dieser Altersgruppe sind insbesondere Frauen betroffen, über dem 50. Lebensjahr haben diese gegenüber Männern ein ca. 7-fach höheres Risiko, eine distale Radiusfraktur zu erleiden. In den meisten Fällen handelt es sich um ein Niedrigenergietrauma, wie etwa ein Stolpersturz auf die ausgestreckte Hand. Besondere Risikofaktoren für das Auftreten von Knochenbrüchen sind eine begleitende postmenopausale Osteoporose oder eine aufgrund von Inaktivität geringe Knochendichte. Die Patienten werden im Alter unsicherer, weniger agil und können Stürze schlechter abfangen.

\section{Ätiologie}

Anatomisch gesehen bildet der distale Radius das radiokarpale sowie radioulnare Gelenk und steht mit der Handwurzel über die kräftigen dorsalen und palmaren extrinsischen Bänder in Verbindung. Er ist dadurch Hauptpfeiler der karpalen Kraftübertragung und nimmt bei einer Traumatisierung ca. $80 \%$ der einwirkenden Kraft auf. Der Processus styloideus radii und die Fossa scaphoidea formen die radiale Säule. Zentral werden die Kräfte über die Fossa lunata und Anteile des distalen Radioulnargelenks aufgenommen; die ulnare Säule umfasst den ulnokarpalen Komplex. Dieser wird durch den triangulären fibrokartilaginären Komplex (TFCC), extrinsische Bänder und die distale Ulna 


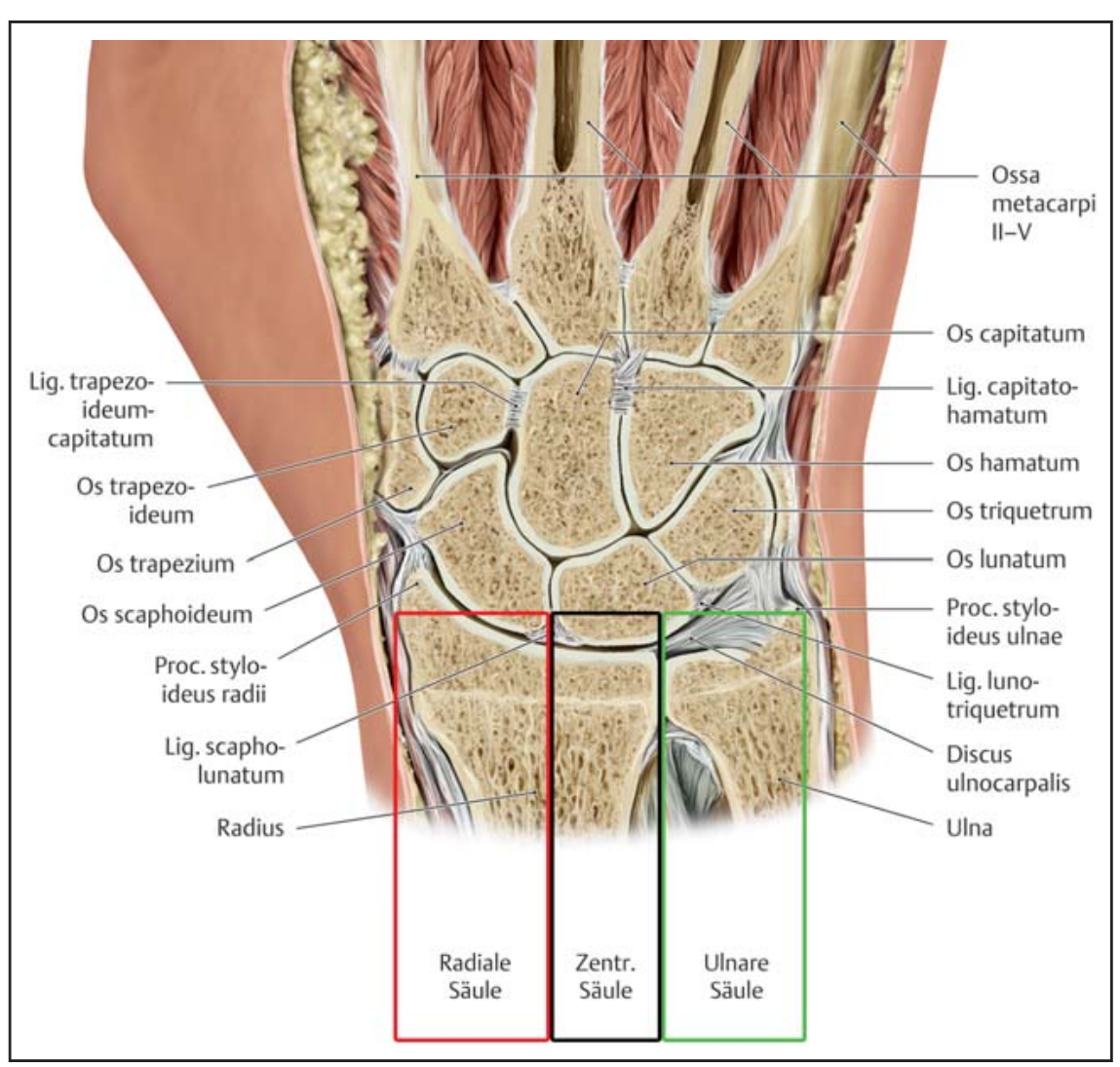

Abb. 1 Anatomie des Handgelenks und Darstellung des 3-Säulen-Modells (Anlehnung an: Schünke M, Schulte E, Schumacher U. Prometheus: LernAtlas der Anatomie. Illustration von M. Voll und K. Wesker. 3. Auflage Stuttgart: Thieme; 2011).

gebildet. Die radiokarpale Gelenkfläche besitzt eine Ulnarneigung mit einem radioulnaren Winkel von ca. $25^{\circ}$ und eine Palmarneigung mit einem dorsopalmaren Winkel von $10^{\circ}$ (Abb. 1 und 2 ).

Die meisten Radiusfrakturen entstehen durch einen Sturz auf die ausgestreckte Hand. Die Stellung des Handgelenks zum Zeitpunkt der Gewalteinwirkung bestimmt ganz erheblich die resultierende Frakturform.

Befindet sich das Handgelenk in einer Streckstellung, so entstehen regelhaft Extensionsfrakturen. Dieser Verletzungsmechanismus findet sich bei $90 \%$ der Frakturen. Befindet sich das Handgelenk zum Unfallzeitpunkt zwischen $40^{\circ}$ und $90^{\circ}$ Extension frakturiert der distale Radius in loco typico etwa 1,5 bis $2 \mathrm{~cm}$ proximal der Radiusgelenkfläche. Bei geringerer Streckung kommt es zu einer Unterarmfraktur, darüber zu Luxationen und Frakturen im Handwurzelbereich. Durch dorsale Dislokation entsteht die typische Gabelstellung des Handgelenks. Häufig ist bei einer Fraktur des Proc. styloideus ulnae der Discus articularis mit verletzt [15] (Abb. 3).
In $10 \%$ ist das Handgelenk zum Unfallzeitpunkt gebeugt, es resultieren dann Flexionsfrakturen. Bei einem Sturz auf den flektierten Handrücken kommt es dagegen zu einer volaren Absprengung.

\section{Diagnostik}

Oftmals erhält der behandelnde Arzt aus der Anamnese des Sturzes bereits Hinweise auf die erlittene Verletzung. Der synkopale Sturz muss vom reinen Stolpersturz differenziert werden. Ebenso sind Begleiterkrankungen $\mathrm{zu}$ erfragen. Ein Bagatelltrauma kann bei begleitender Osteoporose möglicherweise für eine komplexe Unterarm- oder Radiusfraktur ausreichen. Auch neurologische Erkrankungen, ein Karpaltunnelsyndrom und vorausgegangene Verletzungen müssen erfasst werden.

Inspektorisch ist die Deformierung des Handgelenksbereichs sichtbar, es liegt eine schmerzhafte Schwellung und Verbreiterung vor. Durch die Verschiebung der Frakturfragmente kommt es zur typischen Fehlstellung der Hand gegenüber dem Unterarm. Eine Verschiebung zur Streckseite hin wird als Fourchette-

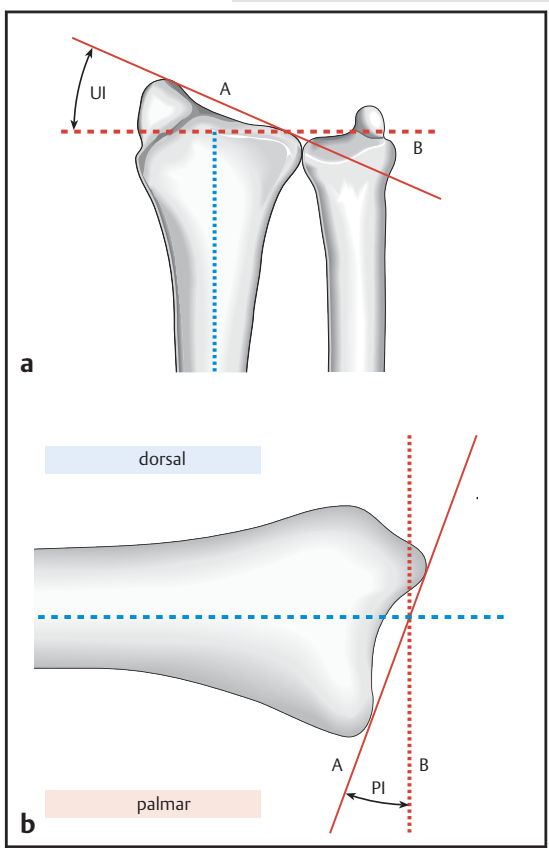

Abb. 2 Bestimmung der ulnaren und palmaren Inklination des distalen Radius [13].

Stellung, die Dislokation des frakturierten Radius zum Daumen hin als Bajonett-Stellung bezeichnet. Bei der klinischen Untersuchung lassen sich Prellmarken, Hämatome, Druckschmerzen und Bewegungsschmerzen feststellen. Die Funktion der angrenzenden Gelenke und der lokale Druckschmerz über dem distalen Radioulnargelenk, über der distalen Ulna und in der Tabatiere sind für differenzialdiagnostische Überlegungen von Relevanz.

Es ist notwendig, die aktive Funktion der Fingergelenke und die Daumenstreckerfunktion zu überprüfen sowie evtl. neurologische Ausfälle und Durchblutungsstörungen zu erkennen. Bei schweren Verletzungen mit höhergradigem Weichteilschaden muss an ein Kompartmentsyndrom gedacht werden.

\section{Bildgebende Diagnostik}

Das Standardverfahren ist die Röntgenaufnahme des Handgelenks in 2 Ebenen. Insbesondere extraartikuläre Frakturformen lassen sich so sicher beurteilen. Bei dem Verdacht auf eine ligamentäre Verletzung mit karpaler Instabilität sind dynamische Untersuchungen unter Durchleuchtung möglich [12] (Abb.4).

Bei bereits einliegendem Osteosynthesematerial ist eine orthograde Darstellung des Gelenkspalts notwendig, um eine 


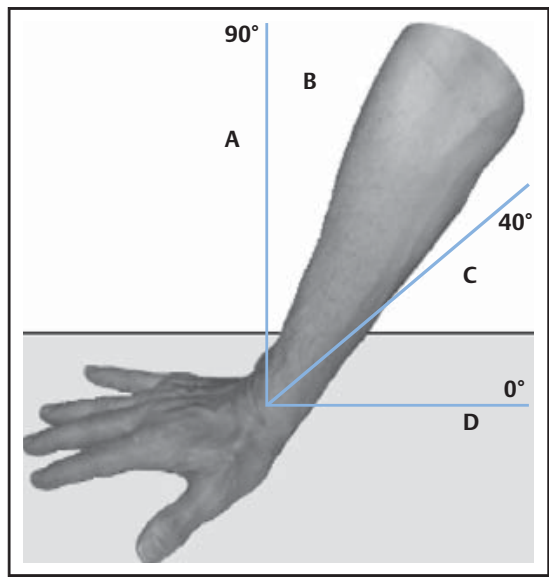

Abb. $\mathbf{3 a}$ bis d Stellung der Hand zum Unfallzeitpunkt und resultierender Frakturtyp: a Luxation/Luxationsfraktur, b Extensionsfraktur, c Unterarmfraktur, d Flexionsfraktur. (aus: Uzdil T, Winker KH. Distale Radiusfrakturen. Orthopädie und Unfallchirurgie up2date $2007 ; 2$ : 1-20) [15].

Überlagerung der Gelenkfläche durch Schrauben- oder Plattenanteile zu vermeiden. Dies lässt sich durch $10^{\circ}$ angehobene Aufnahmen bewerkstelligen.

Liegt ein komplexer, intraartikulärer Frakturverlauf vor, empfiehlt sich eine Analyse des Verletzungsmusters aufgrund der Computertomografie. Mithilfe der CT-Aufnahmen ist eine zuverlässigere Klassifikation der Verletzung möglich, anhand derer die adäquate Therapieform gewählt wird [4]. Knöcherne Begleitverletzungen im Bereich der Handwurzel werden abgebildet, ggf. kann eine 3D-Rekonstruktion für die Therapieplanung hilfreich sein. Bei initial ausgeprägter Fragmentdislokation empfiehlt sich eine CT-Diagnostik nach der Anlage eines Fixateur externe (Abb. 5).

Da zugangsbedingt eine Visualisierung der Gelenkflächen intraoperativ nicht möglich ist, kann die Anwendung eines isozentrischen C-Bogens mit 3-D-Darstellung zusätzliche Informationen liefern. Meier et al. [9] konnten neben einer Optimierung der Fragmentreposition und Schraubenlage eine Verbesserung des postoperativen radiologischen Ergebnisses erzielen. In anderen Studien traten in der Therapiegruppe mit 3D-Darstellung weniger Implantatfehllagen auf [1]. Gerade bei der Verwendung von polyaxial-winkelstabilen Implantaten können einzelne Fragmente nach Reposition exakt adressiert werden. Ob diese zusätzliche Option im späteren klinischen Outcome zu einer Funktionsverbesserung führt, bleibt abzuwarten und

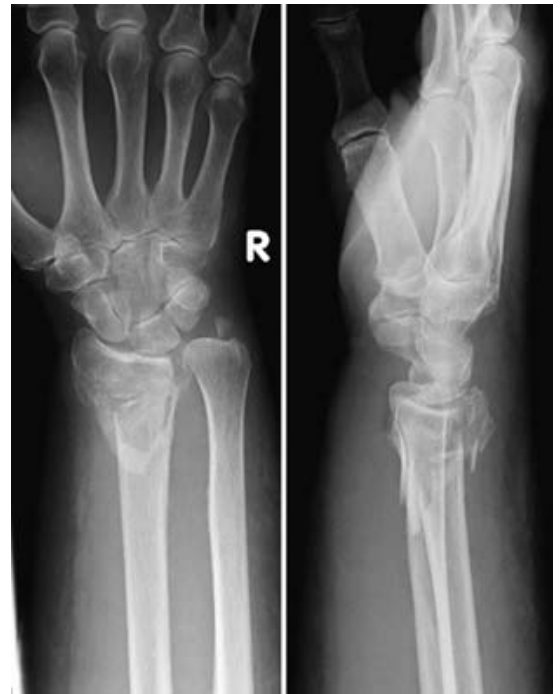

Abb. 4 Beispiel einer distalen Radiusfraktur Typ 23-C2 nach AO.

ist Gegenstand aktueller prospektiver Vergleichsuntersuchungen.

\section{Klassifikation}

Die Differenzierung der Radiusfrakturen anhand einer Klassifikation sollte dem behandelnden Arzt die Beschreibung der Verletzung erleichtern und wesentliche Aspekte erfassen, anhand derer eine Therapieentscheidung getroffen werden kann. Die erste Einteilung der distalen Radiusextensionsfrakturen erfolgte 1783 durch Pouteau und 1814 durch Colles [8]. Die Frakturen werden nach Colles in 4 Klassen unterteilt. Man spricht auch von der ,fractura loco typico sive classico“. Bei dieser Einteilung werden nur Extensionsfrakturen berücksichtigt. Da hierbei der exakte Frakturverlauf oder das etwaige Vorliegen einer mehrfragmentären Bruchform nicht erfasst wird, lässt sich aus der Einteilung kein Behandlungskonzept ableiten (Tab. 1).

Eine weitere Einteilung der Extensionsfrakturen nahm Frykman 1967 vor. Er unterscheidet extra- und intraartikuläre Frakturen mit und ohne Beteiligung des Proc. styloideus ulnae. Diese Einteilung geht nicht darauf ein, ob eine palmare oder dorsale Abkippung vorliegt, da sie sich am posterior-anterioren Strahlengang orientiert. Außerdem geht sie nicht auf Ausdehnung und Richtung einer Dislokation der Frakturfragmente ein. Ebenso werden die dorsale Trümmerzone und die Radiusverkürzung nicht berücksichtigt. Aus der Frykman-Klassifikation

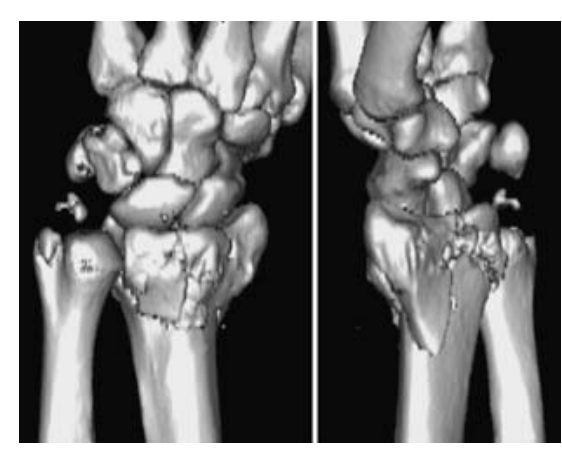

Abb. 5 CT des Handgelenks mit koronarer MPR 3-D-Rekonstruktion.

Tab. 1 Einteilung der distalen Radiusextensionsfrakturen nach Colles [8].

\begin{tabular}{ll} 
Colles I & extraartikulär, nicht disloziert \\
\hline Colles II & extraartikulär, disloziert \\
\hline Colles III & intraartikulär, nicht disloziert \\
\hline Colles IV & intraartikulär, disloziert
\end{tabular}

lässt sich eine Behandlungsindikation nur eingeschränkt ableiten (Abb. 6).

Die Flexionsfrakturen wurden initial 1847 durch Smith beschrieben, er setzt die Lage des dislozierten volaren Kantenfragments in Bezug zur radialen Gelenkfläche. Weiterhin existiert eine Differenzierung der Smith-Frakturen nach Thomas, wobei hier der Verlauf der Frakturlinie von entscheidender Bedeutung ist. Thomas Typ II wird auch als ReversedBarton-Fraktur bezeichnet, bei der es nach volarem Kantenabriss zur vorderen Subluxation des Karpus kommt (Abb. 7).

1984 klassifizierten Melone und Isani die intraartikulären 4-Fragment-Frakturen basierend auf der Analyse radiokarpaler und radioulnarer Schlüsselfragmente [7]. Der Radiusschaft bildet das 1. Fragment, das 2. der Proc. styloideus radii. Das 3. dorsoulnare und das 4. palmar-ulnare Fragment bilden zusammen mit den ligamentären Verbindungen zum Proc. styloideus ulnae und der proximalen Reihe der Handwurzelknochen den sog. medialen Komplex. Die häufigste Frakturform stellen die Typ-II-Frakturen mit Einstauchung des dorsoulnaren Fragments durch das Os lunatum dar (Tab. 2, Abb. 8).

Die einzige Klassifikation neben der AOKlassifikation, die auch Mehrfragmentbrüche kategorisiert und gleichzeitig deren Dislokationsgrad berücksichtigt, ist die Einteilung nach Older [11]. Die Ein- 


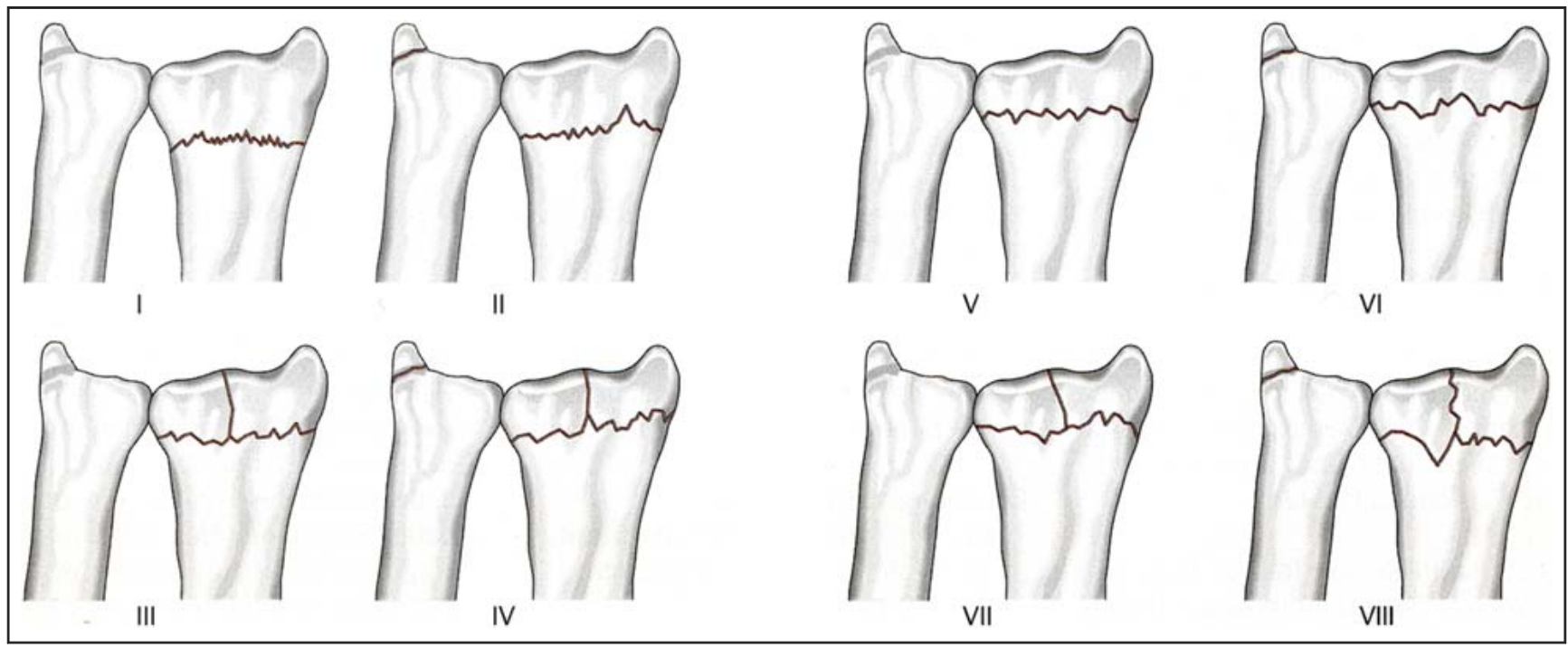

Abb. 6 Klassifikation nach Frykman (1967): Unterteilt wird in extra- und intraartikuläre Frakturen mit und ohne Beteiligung des Proc. styloideus ulnae. Die Komplexität der Fraktur nimmt mit steigender Typenbezeichnung zu [14].

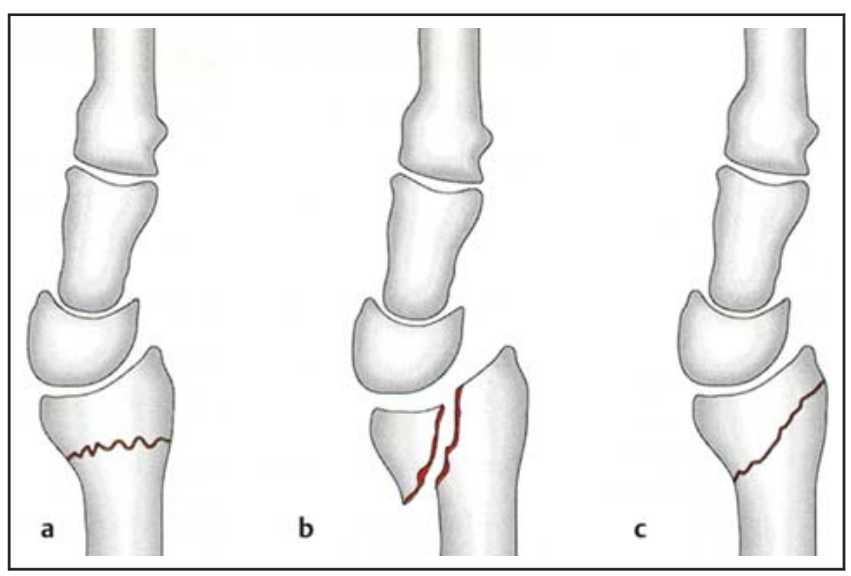

Tab. 2 Frakturklassifikation nach Melone.

\begin{tabular}{ll} 
Typ I & stabil, wenig eingestaucht \\
\hline Typ II & $\begin{array}{l}\text { instabil, medialer Komplex im } \\
\text { Verbund disloziert }\end{array}$ \\
\hline Typ III & $\begin{array}{l}\text { instabil, medialer Komplex im } \\
\text { Verbund disloziert, zusätzliches } \\
\text { volares Schaftfragment }\end{array}$ \\
\hline Typ IV & $\begin{array}{l}\text { instabil, ausgeprägte Dislokation } \\
\text { des dorsalen und volaren Schlüs- } \\
\text { selfragments }\end{array}$
\end{tabular}

teilung erfolgt nach dem Grad der dorsalen Abkippung, der Dislokation, der Radiusverkürzung und dorsaler metaphysärer Einstauchung (Tab.3).

Fernandez [6] bezog bei seiner Klassifikation den Unfallmechanismus mit ein, sodass der Zusammenhang zwischen Pathomechanismus und Frakturmorphologie berücksichtigt wird (Abb. 9). liert sind.
Abb. 7 a bis c Klassifikation der SmithFrakturen: a extraartikuläre Smith-Fraktur mit horizontalem Bruchverlauf (Typ Thomas I). b Intraartikuläre Smith-Fraktur (Typ Thomas II bzw. Reversed Barton). c Extraartikuläre Smith-Fraktur mit schräg verlaufendem Bruchspalt (Typ Thomas III) [14].

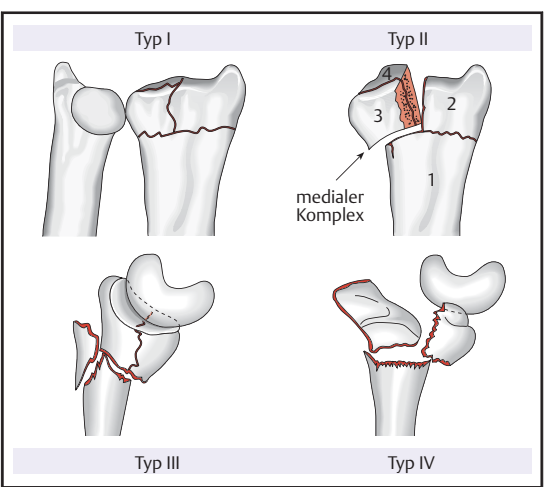

Abb. 8 Die frakturierte Gelenkfläche wird nach Melone in 4 Schlüsselfragmente unterteilt. (1) Radiusschaft, (2) radiales Styloidfragment, (3) dorsoulnares Fragment, (4) palmarulnares Fragment. Die dislozierte Fraktur des medialen Komplexes betrifft sowohl das Radiokarpalgelenk als auch das DRUG und führt zu einer Zerstörung des Gelenkverbunds [14].

\begin{tabular}{ll} 
Typ I & $\begin{array}{l}\text { dorsale Abkippung }<5^{\circ} \text {, Radius- } \\
\text { verkürzung } 7 \mathrm{~mm}\end{array}$ \\
\hline Typ II & $\begin{array}{l}\text { dorsale Abkippung }>5^{\circ} \text {, Radius- } \\
\text { verkürzung } 1-7 \mathrm{~mm}\end{array}$ \\
\hline Typ III & $\begin{array}{l}\text { Abkippung }>5^{\circ} \text {, Radiusverkür- } \\
\text { zung } 4 \mathrm{~mm} \text {, wenig eingestaucht }\end{array}$ \\
\hline Typ IV & $\begin{array}{l}\text { dorsale Abkippung }>5^{\circ} \text {, Radius- } \\
\text { verkürzung neg., deutlich einge- } \\
\text { staucht mit intraartikulärer Be- } \\
\text { teiligung }\end{array}$
\end{tabular}

Neben der Beschreibung der knöchernen Läsion sollte eine Aussage zur Stabilität nach Reposition abgeleitet werden können. Jupiter und Mitarbeiter [8] haben Kriterien abgeleitet, welche regelmäßig zur Stabilitätsbeurteilung herangezogen werden und inzwischen etab-
Als prädisponierend für eine hohe Instabilität wird eine Dorsalabkippung über $20^{\circ}$, die axiale Einstauchung um mehr als $5 \mathrm{~mm}$ und das Vorhandensein einer metaphysären Trümmerzone angesehen. Weitere Instabilitätskriterien sind eine begleitende Fraktur des Proc. styloideus ulnae, eine dislozierte intraartikuläre Fraktur und die radioulnare Instabilität.

\section{AO-Klassifikation}

Im klinischen Alltag ist die Klassifikation der AO (Arbeitsgemeinschaft für Osteosynthesefragen) nach Müller etabliert [10]. 


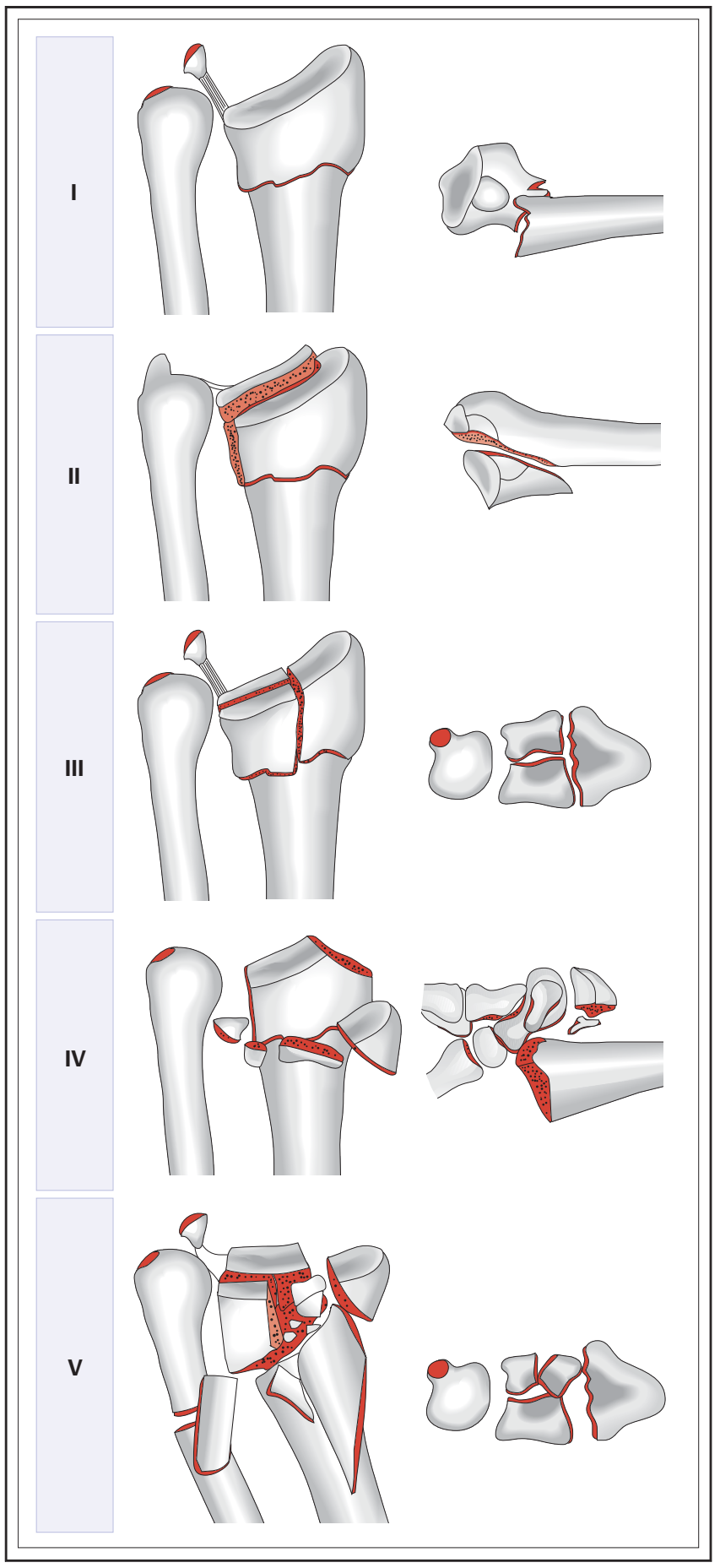

Abb. 9 Klassifikation der distalen Radiusfraktur nach Fernandez. Typ I: Biegungsfraktur: Die Metaphyse kippt in Belastungsrichtung. Typ II: Abscherfraktur: Absprengung einer dorsalen oder palmaren Gelenklippe. Typ III: Kompressionsfraktur: Gelenkflächenbeteiligung mit Einstauchung des subchondralen Knochens. Typ IV: Abrissfraktur: Avulsionsfrakturen und radiokarpale Luxationsfrakturen. Typ V: Kombinationsverletzung: Mischformen aus den Frakturtypen I-IV [14].

Aus der korrekten Einteilung der Fraktur in das Klassifikationsschema nach Müller von 1990 erwachsen direkte Konsequenzen für das therapeutische Vorgehen. Jeder lange Röhrenknochen oder Skelettabschnitt wird mit einer Ziffer versehen und jeder Knochen in 3 Segmente unterteilt.

Man unterscheidet zwischen dem proximalen Segment 1, dem diaphysären
Segment 2 und dem distalen Segment 3. Die Lage der Fraktur ist für die Zuordnung entscheidend. So ergibt sich für den distalen Unterarm die 23. Des Weiteren wird zwischen extraartikulären Bruchformen (Typ A), partiell intraartikulären (Typ B) und komplett intraartikulären Brüchen (Typ C) unterschieden. Jeder Frakturtyp A-C wird weiter differenziert und besteht aus 3 Gruppen mit je 3 Untergruppen. So sind insgesamt 27
Frakturtypen des distalen Unterarms berücksichtigt.

Die Gruppe A1 bilden die isolierten Ulnafrakturen. Die Gruppe A2 beinhaltet einfache extraartikuläre Frakturen ohne Trümmerzone, die Gruppe A3 diejenigen mit Trümmerzone. Die Gruppe B1 beinhaltet Frakturen des Proc. styloideus radii, die sog. Chauffeur-Frakturen. B2Frakturen weisen ein dorsales Kantenfragment auf und werden als BartonFrakturen bezeichnet. Frakturen mit volarem Kantenfragment (Reversed-Barton) bilden die Gruppe B3. Typ C stellen die vollständig artikulären Frakturen dar. Die Gruppe C1 beinhaltet Frakturen mit einfacher artikulärer und metaphysärer Beteiligung, die Gruppe C2 die artikulär einfachen und metaphysär mehrfragmentären Frakturen. Die Gruppe C3 beinhaltet sowohl artikulär als auch metaphysär mehrfragmentäre Frakturen (Abb. 10).

Die AO-Klassifikation erlaubt die Zuordnung einer Fraktur in eine der oben genannten Gruppen anhand von Röntgenaufnahmen in 2 Ebenen. Darüber hinaus lässt sich eine Differenzialindikation für die weitere Therapie stellen, hierauf wird in den nachfolgenden Artikeln dieser Ausgabe differenziert eingegangen.

Die Frakturen der Gruppe A2 sind im Wesentlichen einer konservativen Therapie zugängig, in den letzten Jahren hat sich in der Gruppe der A3-Verletzungen das Therapieregime von einem konservativen Vorgehen zur operativen Versorgung verlagert. Für C-Frakturen kommt je nach Weichteilstatus die primäre Stabilisation mittels Fixateur externe in Betracht, der im Verlauf durch eine winkelstabile Plattenosteosynthese ersetzt wird

\section{Schlussfolgerung}

Um eine Entscheidung für ein adäquates Therapieverfahren treffen zu können, ist es wichtig, das Ausmaß der Verletzung des Radiokarpalgelenks richtig einzuschätzen. Im deutschsprachigen Raum hat sich die Einteilung der handgelenksnahen Unterarmfrakturen anhand der AO-Klassifikation durchgesetzt. Idealerweise ist eine Frakturklassifikation umfassend, eindeutig, anwendbar und überschaubar [16]. Zudem sollten sich prognostische Aussagen über den Heilverlauf ableiten lassen. 
$23-\mathrm{A}=$ Radius/Ulna distal, extraarticuläre Fraktur $23-A_{1}$ $\mathrm{A}_{2}$ $\mathrm{A}_{3}$
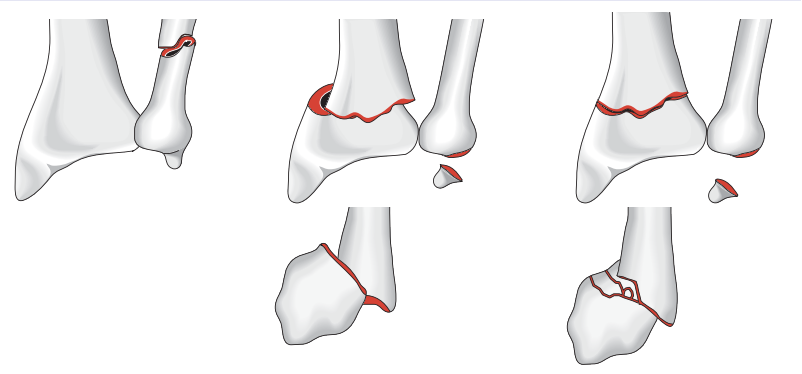

$\mathrm{A}_{3}=$ Radius

$\mathrm{A}_{1}=$ der Ulna, $\mathrm{A}_{2}=$ des Radius,

Radius intakt einfach und impaktiert

mehrfachfragmentär

$23-\mathrm{B}=$ Radius/Ulna distal, partielle Gelenkfraktur des Radius
$23-\mathrm{B}_{1}$

$\mathrm{B}_{2}$
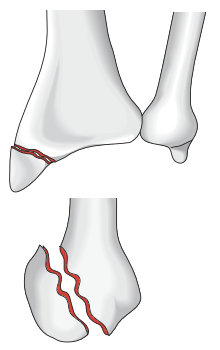

$\mathrm{B}_{1}=$ Sagitalebene

$\mathrm{B}_{2}=$ dorsale Kante (Barton)
$\mathrm{B}_{3}$

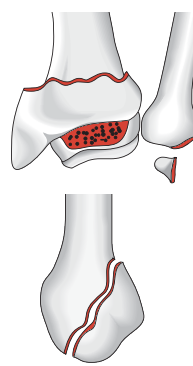

$\mathrm{B}_{3}=$ palmare Kante (reversed Sarton, Goyrand-Smith II)
$23-\mathrm{C}=$ Radius/Ulna, vollständige Gelenkfraktur des Radius

$$
23-C_{1}
$$

$\mathrm{C}_{2}$
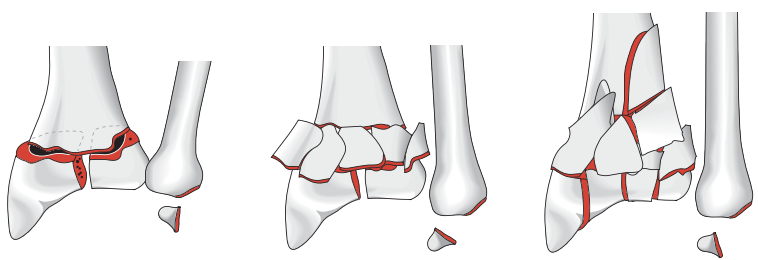

$\mathrm{C}_{3}$

$C_{1}=$ articulär einfach, $C_{2}=$ articulär einfach, $C_{3}=$ mehrfragmentär metaphysär einfach metaphysär mehrfachfragmentär
Abb. 10 AO-Klassifikation nach Müller: 23-A Extraartikuläre Frakturen. 23-B Partielle Gelenkfrakturen. 23-C Vollständig artikuläre Frakturen [14].
${ }^{4}$ Dahlen HC, Franck WM, Sabauri G et al. Fehlklassifikation extraartikulärer distaler Radiusfrakturen in konventionellen Röntgenaufnahmen. Vergleichende Untersuchung der Frakturmorphologie zwischen biplanarer Röntgendiagnostik und CT. Unfallchirurg 2004; 107: 491-498

5 de Putter CE, van Beeck EF, Looman CW et al. Trends in wrist fractures in children and adolescents, 1997-2009. J Hand Surg [Am] 2011; 36: $1810-1815 . e 2$

${ }^{6}$ Fernandez DL, Jupiter J. Fractures of the distal Radius: A practical Approach to Management. New York: Springer;1996

${ }^{7}$ Isani A, Melone jr. CP. Classification and management of intraarticular fractures of the distal radius. Hand Clin 1988; 4: 349-360

8 Jupiter JB. Complex articular fractures of the distal radius: classification and management. J Am Acad Orthop Surg 1997; 5: 119-129

9 Meier R, Geerling J, Hüfner T et al. The isocentric C-arm. Visualization of fracture reduction and screw position in the radius. Unfallchirurg 2011; 114: 587-590

${ }^{10}$ Müller M, Nazarian S, Koch $P$ et al. The AOClassification of Fractures of long Bones. Berlin, Heidelberg, New York, Tokyo: Springer; 1990

11 Older TM, Stabler EV, Cassebaum WH. Colles fracture: evaluation and selection of therapy. J Trauma 1965; 5: 459-476

12 S2-Leitlinie „Distale Radiusfraktur“; Arbeitsgemeinschaft der Wissenschaftlichen Medizinischen Fachgesellschaften, Leitlinien der Deutschen Gesellschaft für Unfallchirurgie e.V. (DGU); Stand: 01.05.2008

${ }^{13}$ Frank J, Pralle H, Marzi I. Funktionelle Anatomie und Biomechanik des Handgelenkes und distalen Radioulnargelenkes. OP-JOURNAL 2003; 19: 4-9

14 Schmidt S, Heinz T, Dávid A. Häufigkeit, Entstehung und Klassifikation der distalen Radiusfraktur. OP-JOURNAL 2003; 19: 10-15

${ }^{15}$ Uzdil T, Winker KH. Distale Radiusfrakturen. Orthopädie und Unfallchirurgie up2date 2007; 2: 1-20

${ }^{16}$ Wittner B, Holz U. General classification of distal radius fractures and treatment of extraarticular distal radius fractures (Type A2 and A3). Chirurg 1993; 64: 880-888

\section{Dr. med. Markus Schubert Oberarzt}

Prof. Dr. med. Andreas Dávid Chefarzt

Helios Klinikum Wuppertal Heusner Straße 40 42283 Wuppertal

Markus.Schubert@Helios-Kliniken.de
Da von keiner Fraktureinteilung allein diese Aspekte in gleicher Weise berücksichtigt werden, sollte die Therapie der distalen Radiusfraktur die Differenzierung zwischen intra- und extraartikulären Frakturen, die Beurteilung von Dislokationen sowie das Vorhandensein von Instabilitätskriterien berücksichtigen und gegebenenfalls ligamentäre und knöcherne Begleitverletzungen adressieren.

\section{Literatur}

${ }^{1}$ Atesok K, Finkelstein J, Khoury A et al. The use of intraoperative three-dimensional imaging (ISO-C-3D) in fixation of intraarticular fractures. Injury 2007; 38: 1163-1169

2 Colles A. Historical paper on the fracture of the carpal extremity of the radius (1814). Injury 1970; $2: 48-50$

${ }^{3}$ Witzel K, Raschka C, Schiffhauer S et al. Epidemiology and chrono-epidemiology of distal radius fractures. Z Orthop Ihre Grenzgeb 2001; 139: 252-255 